\title{
Characterization and integration of oxidation catalysts at small- scale biomass combustion furnaces
}

\author{
Mirjam Matthes ${ }^{1, *}$, Ingo Hartmann ${ }^{1}$, Joachim Schenk ${ }^{2}$, and Dirk Enke ${ }^{3}$ \\ ${ }^{1}$ Deutsches Biomasseforschungszentrum gGmbH, Department Thermo-chemical conversion, 04347 Leipzig, Germany \\ ${ }^{2}$ Hochschule für Technik, Wirtschaft und Kultur Leipzig, Faculty of Mechanical and Energy Engineering, 04277 Leipzig, Germany \\ ${ }^{3}$ Universität Leipzig, Faculty of Chemistry and Mineralogy, 04103 Leipzig, Germany
}

\begin{abstract}
Small-scale biomass combustion is a major part in heat supply from renewable resources. Drawbacks to the environmental background are the pollutant emissions, which are formed as a result of maloperation, suboptimal furnace construction or the biomass fuel composition. The named primary factors can be influenced by several measures, but the achievable emission results are limited. To provide real clean combustion technology with nearly zero pollutant emissions, secondary emission reduction measures are necessary. One of these measures is the application of catalytic flue gas cleaning as integrated or downstream solution. Catalysis is already a state of the art element in many processes and following this, some studies reveal already its potential to reduce $\mathrm{CO}$, VOC as well as particle emissions in small-scale biomass combustion systems. However, a wide application of catalysts in wood combustion units didn't take place so far, because the challenging process conditions demand a proper integration and highly stable and active catalytic materials. For the achievement of well-functioning combustion systems with catalyst solutions a procedure for application-oriented characterization is presented. Initial investigations with commercially available catalysts have shown that the gas hourly space velocity and the oxygen content have the most significant influence on the conversion rate of carbon monoxide and nitrogen oxide. Two samples with different active phases have been compared, one with solely metal oxides and one with metal oxides and noble metals. The one with noble metals showed as expected a higher activity, but also a higher stability.
\end{abstract}

\section{Introduction}

The generation of energy from renewable resources is increasingly in demand to reduce the accumulation of the greenhouse gas carbon dioxide in the atmosphere and accordingly stop global warming. Bioenergy - energy produced out of biomass - provides already $14 \%$ of total energy consumption [1]. The highest share in this sector results from heat production for buildings with traditional and also modern facilities. Biomass combustion in small-scale systems represents here a considerable part. Small combustion furnaces for domestic heating ranging from simple fireplaces in developing countries to modern stoves and pellet boilers in areas like Europe and the United States of America exist all over the world. Dependent on a plurality of parameters like furnace construction, fuel composition and combustion control, a large variety of pollutants is emitted in the flue gas of small-scale biomass combustion systems [2]. Incomplete combustion and unfavourable conditions may lead to significant carbon monoxide (CO), volatile organic compound (VOC), soot, polycyclic aromatic hydrocarbon (PAH) or even dioxin and furan emissions. Beside from organic compounds, other emissions like nitrogen oxides, sulphur oxides or hydrogen chloride can be emitted in relevant concentrations, especially using alternative, agricultural fuels. The emission of the mentioned pollutants reduces the positive environmental effect of the renewable energy generation. In conclusion of this, the legislative regulations for the operation of smallscale furnaces have been tightened during the last years [3, 4, 5]. Therefore, emission reduction measures, primary as well as secondary ones, have to be applied to evolve a truly environmentally friendly energy generation.

\section{Catalysis in small-scale biomass combustion}

\subsection{State of the art in small-scale systems}

The application of catalysts is already established in several industrial processes. We can for example no longer imagine automobiles without a catalyst. The ambition to use also catalysts for emission reduction in small-scale biomass combustion systems increased in the last years with the interest to use renewable energy sources and in parallel growing environmental 
awareness. The main goal by now is to reduce incomplete combusted or formed carbonaceous compounds by integrated or downstream positioned catalysts. Though, first efforts are also have been made to reduce nitrogen oxide emissions by selective catalytic reduction [6].

Suitable catalyst materials for total oxidation of carbon compounds are noble metals as well as mixed metal oxides. In general, noble metals dispersed on alumina supports are common and commercially available, because of their pronounced high activity. Some catalyst solutions for biomass combustion systems are already offered at the market. Basically, there are two ways for the application of catalysts at stoves and boilers. Some manufacturers developed already firing systems with an integrated catalyst [7]. This is favourably, because the facility can be adjusted to the modified pressure and fluid flow conditions with catalyst. By this means comparatively low pollutant emission can be achieved due to optimal construction and catalytic enhanced reaction processes. In the framework of a joint research project for example the constructive optimization and the development of a special down-draft wood log stove accompanied by the development and integration of catalytic devices has been carried out $[8,9]$. The other business branch comprises retrofitting of already operating systems. Here are also several solutions at the market by now $[10,11,12]$. However, there is only rare documentation of the long-term stability and no comprehensive analysation of the behaviour from the used catalysts during the manifold conditions occurring in stoves and boilers.

The major part of described research in literature deals with the effect of catalysts at single-room heaters [9, 1320]. Only some results have been presented from boilers with catalysts [21-23]. The batch-combustion, carried out in furnaces using wood logs as fuel, results in strongly fluctuating conditions. The flue gas composition as well as the temperature varies in the course of one burning cycle. The conditions in pellet boilers are more stable during regular operation, but here also differing states like start and burnout phases or part load operation occur. There the highest pollutant concentrations are emitted and the catalytic effectivity is most important. Most of the described samples consisted of platinum and palladium on ceramic or metallic support materials often with an $\gamma-\mathrm{Al}_{2} \mathrm{O}_{3}$-washcoat. In contrast to that, Bensaid et al. [16] and Carnö et al. [17] analyzed lanthanumchromium-perovskites and copper-manganese-oxides. In general, noble metals show a significant higher oxidation activity, though metal oxides can be beneficial with respect to their stability under some conditions. The direct comparison of a platinum with a coppermanganese-oxides containing catalyst showed a considerably higher activity for carbon monoxide and volatile organic compounds oxidation of the noble metal version [17].

\subsection{Challenges and chances for emission reduction with state of the art catalysts}

In the first place, investigations on the reduction of carbon monoxide and volatile organic compounds by oxidation catalysts are pursued and described. Even though the catalytic oxidation of carbon monoxide can already take place at room temperature [24], the so far at biomass combustion systems applied catalysts show a considerable reduction at temperatures above 250$300{ }^{\circ} \mathrm{C}[14,20,23]$. Beside a preferably low reaction temperature the catalysts in small-scale stoves and boilers also have to feature a wide range and frequent changes in temperature. If the catalyst is applied downstream of a stove, temperatures between 250 and $500{ }^{\circ} \mathrm{C}$ can be present $[14,16,20]$. When the catalyst position is inside the furnace, the temperature can rise up to $750-850{ }^{\circ} \mathrm{C}[9,17,22]$, dependent on the distance to the flame. Since the highest pollutant emissions are generated during start and burnout phases, when the temperatures are low, catalyst heating systems are sometimes applied to gain higher pollutant reduction [14, 21-23]. Literature data show that catalytic conversion rates vary in a wide range and can be hardly compared because of the multitude of differences in the process conditions and mostly not precisely described catalyst probes. In general, the reduction rates for carbon monoxide are clearly higher than for VOC. The often applied noble metal catalysts based on platinum and palladium provide a high activity for $\mathrm{CO}$ oxidation. With a good dimensioned and active catalyst reduction rates over $90 \%$ can be realized $[19,20,21]$. Nevertheless, very high pollutant concentrations $[13,18]$, catalyst bypassing due to leakages [15] or insufficient process temperatures [23] can restrict the performance of the applied catalysts. The reduction rates for VOC are often lower than the rates for $\mathrm{CO}$ or at most equal. The compound methane, which has usually the highest share in VOC flue gas concentration from biomass combustion systems $[14,25]$, is most difficult to oxidize. In this context, a high conversion rate for all VOC except methane [17] and also a fast deactivation of catalysts solely for the oxidation of methane [19] has been observed.

Beside the reduction of gaseous pollutants by catalysis also particulate matter (PM) can be removed from the flue gas. Different mechanisms have to be taken into account. Soot or carbonaceous particle precursors can be catalytically oxidized and accordingly the particle amount in the flue gas is reduced. Particles, organic as well as inorganic ones, can furthermore be deposited at the catalyst surface. As a result the PM concentration is reduced, but also the catalytic active surface is covered and accordingly a deactivation takes place. This is especially critical when alternative non-wood fuels with a higher particle formation are combusted [23]. Nevertheless, several described research results with single-room heaters have shown that a reduction of 30 $50 \% \mathrm{PM}$ is possible $[13,19,20]$.

An important issue for the use of catalysts in practical operation is their long-term stability. Only a few research investigations on this topic are published by 
now $[9,19,20,23]$. It has been observed that the activity and stability of the applied catalysts can vary dependent on the existing boundary conditions. Furthermore, maintenance of the catalyst during long-term operation is necessary. Because of the dependency of the catalytic process from various conditions, the aimed pollutant reduction is often not achieved in practice or only for a limited operation time. Also, during regular operation of small-scale combustion systems temperature and flue gas composition vary to a certain extend. A detailed characterization of catalyst activity and a proper integration is necessary to ensure the intended effectivity and long-term stability.

\section{Application-related characterization and integration of catalysts}

\subsection{Essential boundary conditions}

The following main boundary conditions have to be mentioned in conjunction with catalysis at small-scale biomass combustion systems: gas composition, temperature, pressure and fluid flow conditions. The integration of a catalyst, especially close to the combustion chamber, may influence the initial conditions and can as a result change the combustion process. In Fig. 1 the sequence of combustion and catalysis with its boundary conditions and interdependencies is presented as schematic view.

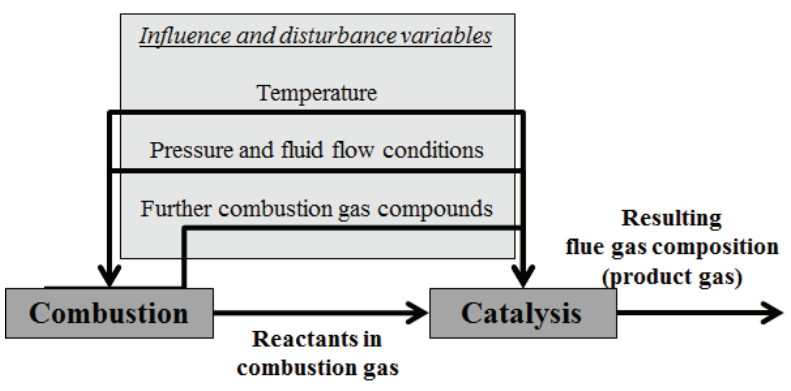

Fig. 1. Catalytic process in biomass combustion systems with boundary conditions and interdependencies.

In general, dependent on the position in the combustion systems the temperature ranges from maximum values between 800 and $1200{ }^{\circ} \mathrm{C}$ to values between 100 and $350{ }^{\circ} \mathrm{C}$ at the outlet of the furnace. Either by natural or induced draft the combustion takes usually place having a negative pressure in the combustion chamber, which has a level of $15-30 \mathrm{~Pa}$.

The pressure drop of a used catalyst has to be very low to prevent an unfavorable or even security-relevant effect on the combustion process. Systems operating with a chimney fan have a higher tolerance than those working with natural draft.

Beside the static pressure, the catalyst changes also the fluid flow conditions in the furnace. This may induce a positive or negative effect on the occurring reactions.

The main flue gas components resulting of an almost total combustion of biomass are nitrogen, carbon dioxide, water and oxygen. Because of partial incomplete combustion smaller or larger quantities of carbon monoxide, volatile up to semi-volatile organic compounds as well as soot are formed. Apart from these components several other elements are present in the fuel, in which the concentration varies according to the fuel type.

A relevant influence on the flue gas composition have the following elements: $\mathrm{N}, \mathrm{K}, \mathrm{P}, \mathrm{Ca}, \mathrm{Mg}, \mathrm{S}, \mathrm{Cl}$. In dependence of several process parameters, as for example temperature and air supply, the flue gas components $\mathrm{SO}_{2}, \mathrm{NO}_{\mathrm{X}}, \mathrm{PCDD} / \mathrm{F}, \mathrm{HCl}$ and ash occur.

\subsection{Characterization procedure}

A comprehensive catalyst utilization in different singleroom heaters as well as central heating furnaces can only be achieved by an universal catalyst characterization for the application. So far, the integration of catalysts in combustion systems was based largely on trial and error. Several optimization loops are necessary, for example when the catalyst usage conditions are not met in all operation phases or changes in the construction of the furnace are necessary to maintain good combustion conditions with the additional pressure drop of an integrated catalyst.

A detailed characterization of the catalyst usage conditions as well as an analysis of the catalyst monolith induced process changes can minimize further following expenses for the integration in special stoves and boilers. In conclusion of these considerations, a procedure for the characterization of promising catalysts for small-scale biomass combustion systems has been developed to examine stepwise the effectivity and also the operation range. The general procedure is shown in Fig. 2

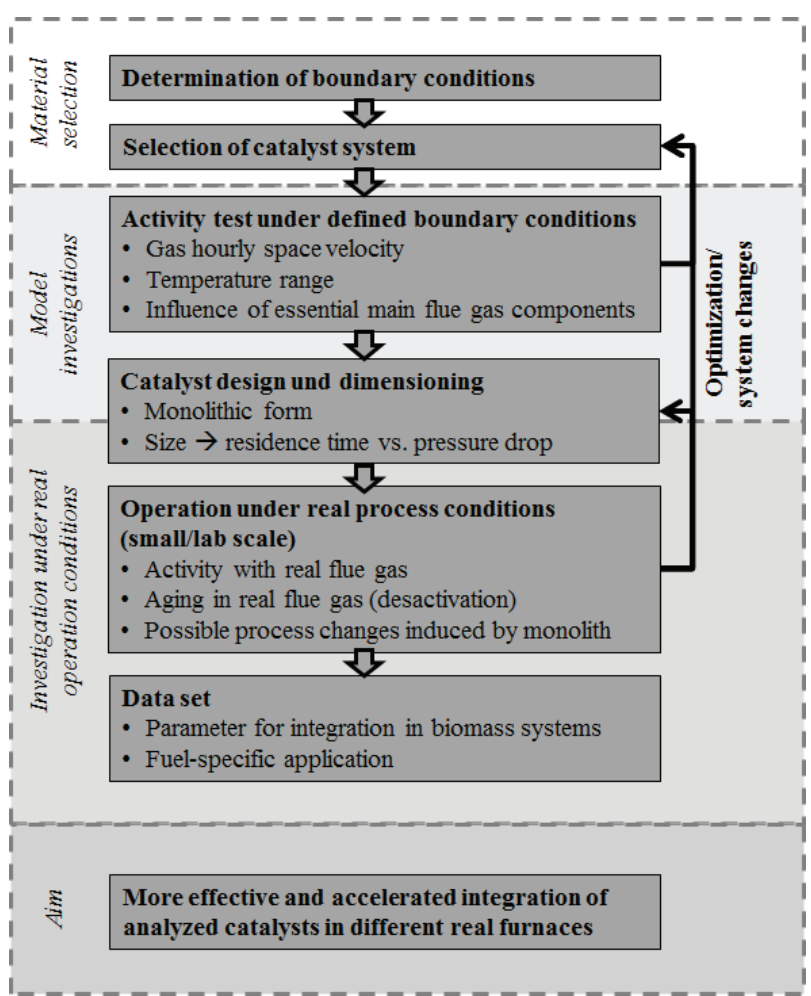

Fig. 2. Characterization procedure for catalyst application in small-scale biomass combustion systems. 
The procedure is realized with two laboratory facilities. In the first one the activity of the catalyst probes for selected pollutants is analyzed with a model gas mixture containing basic flue gas components. The facility allows an analysis of the operation range of the catalyst probe and if this range covers the operation phases of biomass combustion systems. In Fig. 3 the facility is shown as a scheme. The main flue gas components for biomass combustion on a quantity basis are nitrogen, carbon dioxide, oxygen and water. These four compounds are the basis of the model gas. All other elements have a clearly lower concentration and their amount strongly depends on the conditions of the combustion process. In general, incomplete oxidized carbon compounds as $\mathrm{CO}$ and $\mathrm{VOC}$, nitrogen oxides and particulate matter are the most relevant compounds in the lower concentration range.

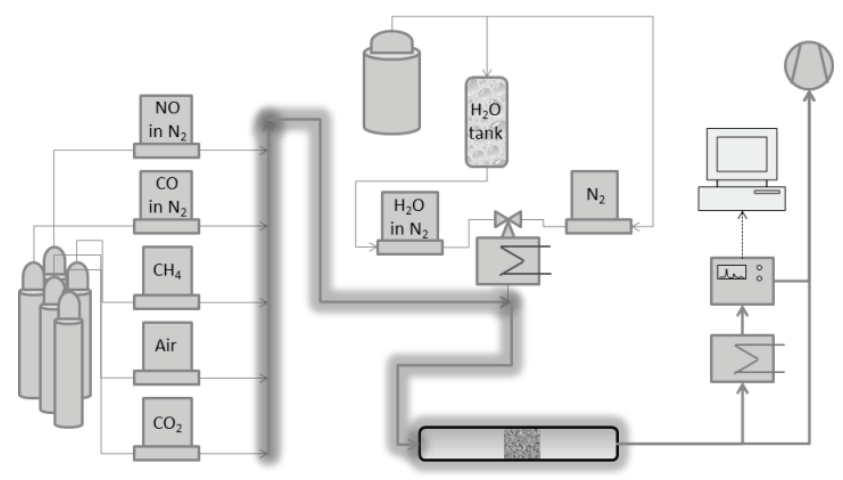

Fig. 3. Scheme of laboratory facility for characterization with model gas.

Design of Experiments (DoE) methods are used to determine the catalyst operation range considering the concentration range of the flue gas components. The gas concentrations are analyzed with an infrared and a paramagnetic oxygen analyzer (type EL 3020, ABB). The influence of particulate matter is though not examined on the model gas level by now.

The real flue gas conditions and at the same time the influence of PM on the catalyst activity are analyzed with a second laboratory facility. The combustion of comminuted biomass is realized on a very small scale with a furnace having a nominal heat output of $1-2 \mathrm{~kW}$. A scheme of the facility is shown in Fig. 4. The construction according to the downdraught combustion principle with mass flow controllers for air supply and a dosage system for the supply of comminuted biomass allows the defined adjustment of different combustion states.

The characterization of the catalyst performance and the influences of the monolith on the combustion process are carried out by comparative operation of the facility with and without catalyst. The flue gas composition as well as temperature and pressure at different positions are measured continuously.

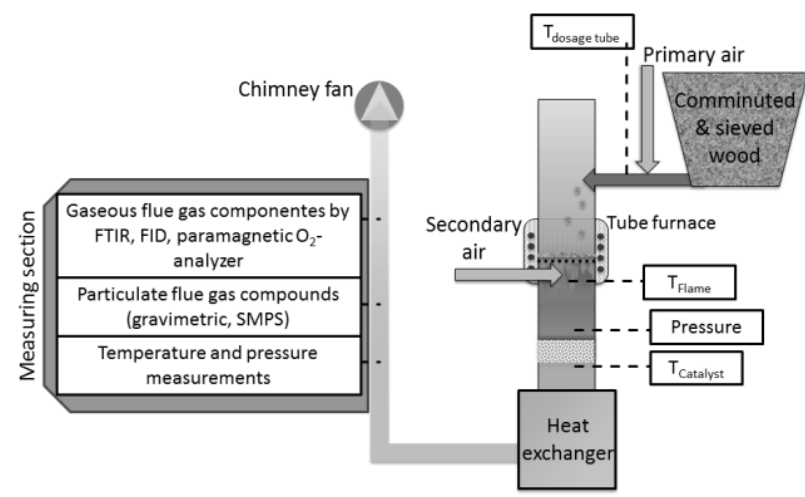

Fig. 4. Scheme of laboratory facility for characterization under real combustion conditions ( $\mathrm{T}$ - temperature; FTIR - Fourier transform infrared spectroscope; FID - Flame ionization detector; SMPS - Scanning mobility particle sizer).

\subsection{Initial findings by model gas investigations}

Some commercially available catalyst probes have been tested to check their applicability for the use in biomass combustion furnaces. The following results have been determined with two samples having both an $\alpha$-alumina foam as support and a high-temperature stable $\gamma$-alumina washcoat. One probe has a solely metal oxide active phase. The active phase of the other one is out of metal oxides and noble metals. The precise composition is not disclosed by the manufacturer. The probes have been tested in a model gas based on a potential flue gas composition of combustion furnaces. The following three steps have been performed. At first, the probes have been heated twice to $700{ }^{\circ} \mathrm{C}$ with corresponding cooling phases. Afterwards they have been heated to $500{ }^{\circ} \mathrm{C}$ for five hours, while the flue gas concentration has been varied according to determine influences of several gas components on the conversion rate by DoEmethods. In the final third step the probes have been heated again twice to $700{ }^{\circ} \mathrm{C}$. As model gas for the start and final test the following composition has been set: 15 Vol.- $\% \quad \mathrm{CO}_{2}, \quad 8$ Vol.- $\% \quad \mathrm{H}_{2} \mathrm{O}, \quad 5.5$ Vol.- $\% \quad \mathrm{O}_{2}$, $1700 \mathrm{ppm} \mathrm{CO}, 175 \mathrm{ppm} \mathrm{CH}_{4}$ and 100 ppm NO. CO and $\mathrm{CH}_{4}$ have been chosen as representative for the gaseous incomplete oxidized pollutants in the flue gas. No catalytic conversion has been observed for methane using the described catalyst samples. The CO conversion differed according to the used probe and also dependent on the period of use. The temperature-conversion-curves are shown in Fig. 5 for both catalysts during the start and final step of the model gas tests.

The sample with noble metals showed as expected a clearly higher conversion rate and also a lower light-off temperature. Furthermore, a conclusion about the stability of the probes has been visible.

The probe with noble metals remained stable with certain variations during the model gas characterization. 


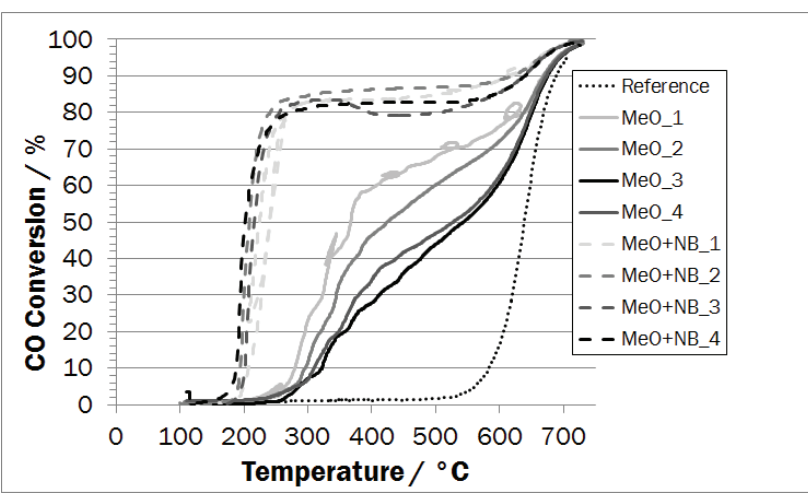

Fig. 5. Carbon monoxide conversion of two commercially available catalyst probes - one with a metal oxide (MeO) active phase and one with a phase composed of metal oxides and noble metals $(\mathrm{MeO}+\mathrm{NB})$.

The activity of the probe with the solely metal oxide active phase decreased considerably during the first and second step of the model gas experiments. In the third step ( $3^{\text {rd }}$ and $4^{\text {th }}$ heating run, see Fig. 5) the sample has been stabilized in all probability, because the conversion rate of the fourth run was slightly higher than the third one.

The second step of the model gas characterization has been carried out by variation of the parameters shown in Table 1 using two levels. The levels have been defined based on conditions occurring during real operation. The temperature and the $\mathrm{CO}_{2}$ concentration have been kept constant at $500{ }^{\circ} \mathrm{C}$ and 12.0 vol.- $\%$ respectively.

Table 1. Parameter and levels for step two of model gas characterization

\begin{tabular}{|c|c|c|}
\hline Parameter/Level & $\mathbf{1}$ & $\mathbf{2}$ \\
\hline $\mathrm{CO} / \mathrm{CH}_{4}[\mathrm{ppm}]$ & $300 / 160$ & $1000 / 175$ \\
\hline $\mathrm{O}_{2}$ vol.- $\%$ & 3.0 & 14.0 \\
\hline $\mathrm{NO}[\mathrm{ppm}]$ & 100 & 300 \\
\hline $\mathrm{H}_{2} \mathrm{O}[\mathrm{vol} .-\%]$ & 5.0 & 10.0 \\
\hline $\mathrm{GHSV}\left[\mathrm{h}^{-1}\right]$ & 50,000 & 100,000 \\
\hline
\end{tabular}

For evaluation of the data the effects of each parameter has been determined with the following equation:

$$
\text { Effect }=\bar{y}_{\text {level } 1}-\bar{y}_{\text {level } 2}
$$

The average value of the results with level 1 of one parameter is subtracted with the average value of level 2 . The calculated conversion rate has been chosen for evaluation of the effects. The analysis of the reference data showed that a standard deviation of $2-3 \%$ exists for the concentration of the gas components $\mathrm{CO}, \mathrm{CH}_{4}$ and NO. Therefore, small conversion changes can't be detected.
Table 2. Average conversion rates for both $\mathrm{CO} / \mathrm{CH}_{4}$ levels and effects (change of conversion) for $\mathrm{O}_{2}$ and GHSV changes.

\begin{tabular}{|c|c|c|c|c|c|}
\hline $\begin{array}{c}\text { Values } \\
\text { in \% }\end{array}$ & & $\begin{array}{c}\mathbf{C O} / \mathbf{C H}_{\mathbf{4}} \\
\text { level 1 }\end{array}$ & $\begin{array}{c}\mathbf{C O} / \mathbf{C H}_{\mathbf{4}} \\
\text { level 2 }\end{array}$ & $\begin{array}{c}\text { Effect } \\
\mathbf{O}_{\mathbf{2}} \downarrow\end{array}$ & $\begin{array}{c}\text { Effect } \\
\mathbf{G H S V} \downarrow\end{array}$ \\
\hline \multirow{2}{*}{$\mathrm{MeO}$} & $\mathrm{CO}$ & 40.1 & 40.1 & 1.4 & 15.6 \\
\cline { 2 - 6 } & $\mathrm{NO}$ & 13.6 & 12.6 & -9.1 & 2.6 \\
\hline \multirow{2}{*}{$\begin{array}{c}\mathrm{MeO} \\
+\mathrm{NB}\end{array}$} & $\mathrm{CO}$ & 69.0 & 71.8 & 3.5 & 14.9 \\
\cline { 2 - 6 } & $\mathrm{NO}$ & 9.5 & 6.2 & -7.0 & 1.7 \\
\hline
\end{tabular}

In Table 2 some data for the clearest influences are given. The results revealed that the change in gas hourly space velocity (GHSV) induced the most significant change of the conversion rate in the analysed parameter range. The $\mathrm{CO}$ conversion was $14.9 \%$ and $15.6 \%$ higher with the lower GHSV. Furthermore, the change of the $\mathrm{O}_{2}$ concentration from 14.0 to 3.0 vol.- $\%$ induced a decrease in $\mathrm{NO}$ conversion. The $\mathrm{CO}$ conversion increased in contrast. Previous measurements with an FTIR analyser showed that NO is converted to $\mathrm{NO}_{2}$. The reduced conversion rate of $\mathrm{NO}$ with lower oxygen content can be influenced by two effects. A lower availability of oxygen can result in a lower conversion rate. Furthermore, $\mathrm{NO}_{2}$ formed by the oxidation of $\mathrm{NO}$ can act as oxidizing agent for example for the conversion of CO. Oxidation with $\mathrm{NO}_{2}$ and accordingly back reaction to $\mathrm{NO}$ increases potentially with decreasing $\mathrm{O}_{2}$ concentration. Beside those general tendencies for both catalyst materials also a difference between the catalyst with and without noble metals was visible. The catalyst with noble metals showed an increased conversion rate with a higher concentration of $\mathrm{CO} / \mathrm{CH}_{4}$. In contrast to this, the NO conversion rate decreased. For the solely metal oxide catalyst only a small decrease of NO conversion appeared. The effects for $\mathrm{H}_{2} \mathrm{O}$ and $\mathrm{NO}$ changes have been negligible as well all effects on $\mathrm{CH}_{4}$ conversion.

Experiments with the same catalyst systems used during model gas analysis are currently carried out in the laboratory facility shown in Fig. 4 under real gas conditions. The evaluation of the data in comparison with the model gas analysis aims to reveal the effect of further flue gas components on stability, especially the influence of particulate matter.

\section{Conclusions}

Catalysts are potentially a useful device in small-scale biomass combustion systems to reduce the pollutant emissions to a lower level. They are able to reduce a wide variety of pollutants for example soot, hydrocarbons and carbon monoxide. Some research results show already that a reduction, especially of carbon monoxide, is possible also over several weeks of operation. Nevertheless, the demands on activity and 
stability for pollutant reduction are high. An appropriate analysis and characterization of suitable catalyst materials is therefore necessary to promote their effective integration and accordingly their acceptance and utilisation. A promotion of use-oriented catalyst analysis in the case of small-scale biomass combustion is intended by the presented characterization methods. A deactivation of catalysts has been observed in different cases. Investigations described in literature with real combustion systems revealed already the negative effect of particles deposited on the catalyst surface. In the described model gas experiments no particles have been present. Therefore, the deactivation of the metal oxide sample without noble metals can be assigned to thermal effects or the influence of a gaseous component. No visual apparent loss of material has been observed.

The described research work was amongst others supported by funds of the Federal Ministry of Food and Agriculture (BMEL) based on a decision of the Parliament of the Federal Republic of Germany via the Federal Office for Agriculture and Food (BLE) under the innovation support programme.

\section{References}

1. REN21, 2016, Renewables 2016 Global Status Report (Paris: REN21 Secretariat), ISBN 978-39818107-0-7

2. S. Ozgen, S. Caserini, S. Galante, M. Giugliano, E. Angelino, A. Marongiu, F. Hugony, G. Migliavacca, C. Morreale, Atmos. Environ. 94 (2014)

3. J. Villneuve, J.H. Palacios, P. Savoie, S. Godbout, Bioresour. Technol. 111 (2012)

4. United States Environmental Protection Agency, FACT SHEET: Summary of Requirements for Woodstoves and Pellet Stoves, https://www.epa.gov/residential-wood-heaters/factsheet-summary-requirements-woodstoves-andpellet-stoves (accessed online April 2017)

5. European Environmental Bureau (EEB), Air \& domestic heating, Gasping for Air - Twelve Factsheets on Air Pollution in the EU, http://www.eeb.org/index.cfm/library/?firstpublicati ons $=1 \&$ month $=0 \&$ Air $=1 \&$ year $=0$ (accessed online April 2017)

6. M. Matthes, M. König, I. Hartmann, Kombinierte Minderung von Staub und Stickoxiden an Biomassefeuerungen, Proceedings of 8 . AbscheiderFachgespräch ,Partikelabscheider in Biomassefeuerungen " (Straubing, 2017)

7. United States Environmental Protection Agency, List of EPA Certified Wood Stoves, https://www.epa.gov/compliance/list-epa-certifiedwood-stoves (accessed online April 2017)

8. DBFZ Deutsches Biomasseforschungszentrum gemeinnützige $\mathrm{GmbH}$, Novel low-emission stove (DBU-NEKO) - press release, https://www.dbfz.de/en/focus-areas/referenceprojects/novel-low-emission-stove-dbu-neko.html (Access online April 2017)
9. R. Bindig, S. Butt, I. Hartmann, D. Dvoracek, W.-D. Einicke, D. Enke, B. Specht, F. Werner, DBFZ Report Nr. 27, Neuartiger emissionsarmer Kaminofen (DBU-NEKO) (Leipzig, 2016) ISBN: 978-3-946629-05-4

10. Firecat, http://www.firecatcombustors.com/ (Accessed online April 2017)

11. DR PLEY ENGINEERING \& CONSULTING (BUCHAREST) SRL, ChimCat ${ }^{\circledR}$ OXI, http://drpley.com/en/imprint/

12. Blue Fire $\mathrm{GmbH}$, The catalyst for stoves and small heating systems, http://blue-fire.org/en/

13. A. Hukkanen, T. Kaivosoja, O. Sippula, K. Nuutinen, J. Jokiniemi, J. Tissari, Atmos. Environ. 50 (2012)

14. F. Ozil, V. Tschamber, F. Haas, G. Trouvé, Fuel Process. Technol. 90 (2009)

15. R. Mack, H. Hartmann, Performance of catalytic and non-catalytic foam ceramic elements in log wood stoves, Proceedings of 24th Eur. Biomass Conf. and Exhibition (Amsterdam, 2016)

16. S. Bensaid, F.A. Deorsola, D. Fino, N. Russo, Catal. Today 197 (2012)

17. J. Carnö, M. Berg, S. Järås, Fuel 75, 959-965 (1996)

18. T. Kaivosoja, A. Virén, J. Tissari, J. Ruuskanen, J. Tarhanen, O. Sippula, J. Jokiniemi, Chemosphere $\mathbf{8 8}$ (2012)

19. R. Bindig, S. Butt, I. Hartmann, M. Matthes, C. Thiel, Catalysts 2 (2012)

20. M. Matthes, I. Hartmann, Chem. Eng. Technol. 40, 2 (2017)

21. Y. Roy, M. Lefsrud, V. Orsat, F. Filion, J. Bouchard, Q. Nguyen, L.-M. Dion, A. Glover, E. Madadian, C. P. Lee, Biomass Bioenerg. 66 (2014)

22. M. Ferrandon, M. Berg, E. Björnbom, Catal. Today 53 (1999)

23. M. Matthes, I. Hartmann, A. Groll, U. Riebel, Biomass Conv. Bioref. 6 (2016)

24. M.-G. Jeong, I. H. Kim, S. W. Han, D. H. Kim, Y. D. Kim, J Mol. Catal. A-Chem. 141 (2016)

25. M. Olsson, Residential biomass combustion emissions of organic compounds to air from wood pellets and other new alternatives, Thesis for the degree of doctor at Chalmers Uni. Technol. (Göteborg, 2006) ISSN 0346-718X 